\title{
MANEJO DE LA EXTRACCIÓN DENTAL EN PACIENTES SOMETIDOS A TERAPIA ANTICOAGULANTE: UNA REVISIÓN DE LA LITERATURA.
}

\author{
MANAGEMENT OF DENTAL EXTRACTION IN PATIENTS UNDER \\ ANTICOAGULANT THERAPY: A LITERATURE REVIEW.
}

\author{
Jessica Micaela Yamunaqué-Vire ${ }^{1}$, Freddy Merchán-Reyes ${ }^{1}$, Paúl Fernando Guillén-Guerrero ${ }^{2}$ \\ ${ }^{1}$ Estudiante de la Facultad de Odontología de la Universidad de Cuenca. Ecuador. \\ ${ }^{2}$ Docente de la Facultad de Odontología de la Universidad de Cuenca.Ecuador.
}

\begin{abstract}
Resumen
En los últimos años la extracción de piezas dentales en pacientes que consumen anticoagulantes ha aumentado, y con ello también la necesidad de nuevos estudios que nos permitan conocer cómo abordar dichos casos. La terapia anticoagulante oral se utiliza para prevenir enfermedades de tipo tromboembólico. Entre los anticoagulantes tenemos los Antagonistas de la vitamina $\mathrm{K}$ y los Anticoagulantes directos orales. El objetivo de este estudio es analizar el tipo de manejo que necesita un paciente anticoagulado, además de las limitaciones, indicaciones y medidas hemostáticas para la extracción dental en estos pacientes. Se realizó una búsqueda en las bases digitales de Pubmed, Science Direct, Cochrane y SciELO, obteniendo un total de 2176 artículos que luego de la implementación de criterios de inclusión y exclusión dio como resultado 59 artículos para esta revisión bibliográfica. El manejo en estos pacientes debe realizarse con suma cautela tomando en cuenta su estado sistémico, se realizará la extracción de manera segura sin suspensión ni modificación del medicamento, cuando se ha planificado la intervención quirúrgica con una técnica atraumática de una a tres piezas y el INR es $\leq 3.5$. Es fundamental para el odontólogo tener el conocimiento actualizado del trato a pacientes anticoagulados y sus complicaciones hemorrágicas.
\end{abstract}

Palabras clave: Warfarina, Anticoagulantes, Anticoagulante oral directo, Extracción dental, Cirugía oral.

\begin{abstract}
In recent years the teeth extraction in patients who consume anticoagulants has increased, and with it also the need for new studies that allow us to know how to address such cases. Oral anticoagulant therapy is used to prevent thromboembolic type diseases. Among anticoagulants we have Vitamin K Antagonists and direct oral anticoagulants. The objective of this study is to analyze the type of management that an anticoagulated patient needs, in addition to the limitations, indications, and hemostatic measures for dental extraction in these patients. A search was made in the digital databases of Pubmed, Science Direct, Cochrane and SciELO, obtaining a total of 2176 articles that after the implementation of inclusion and exclusion criteria resulted in 59 articles for this literature review. The management in these patients should be done with extreme caution taking into account their systemic state, the extraction will be done safely without suspension or modification of the drug, when the surgical intervention has been planned with an atraumatic technique of one to three pieces and the INR is $\leq 3.5$. It is essential for the dentist to have updated knowledge of the treatment of anticoagulated patients and their bleeding complications.
\end{abstract}

Key words: Warfarin, Anticoagulants, Direct oral anticoagulant, Dental extraction, Oral surgery.

\section{Introducción}

La extracción dental es un procedimiento quirúrgico frecuente, por esta razón, el odontólogo debe estar preparado para atender a cualquier paciente que se encuentre sistémicamente sano o enfermo, y así evitar el riesgo de complicaciones provocadas por las intervenciones odontológicas o efectos secundarios de la medicación que reciben. ${ }^{1,2}$ Los anticoagulantes orales son utilizados 
por pacientes que padecen diferentes enfermedades como cardiopatías isquémicas, trombosis venosa profunda, infarto de miocardio, embolias pulmonares, válvulas cardíacas artificiales y fibrilación auricular, también se administran en pacientes con accidentes cerebrovasculares o cirugía de bypass, con el objetivo de evitar eventos tromboembólicos. ${ }^{3-6}$

Los anticoagulantes orales se clasifican según su mecanismo de acción en Antagonistas de la vitamina $\mathrm{K}$ (AVK), Inhibidores directos de la trombina e inhibidores directos del factor $\mathrm{Xa}$, estos dos últimos conocidos como anticoagulantes directos orales (ACDO) ${ }^{4,6-8}$

Los AVK actúan inhibiendo los factores de coagulación dependientes de la vitamina K (II, VII, IX, X), dentro de este grupo se encuentra la warfarina y el acenocumarol6. Estos se caracterizan por tener un inicio de acción lento, su efecto tarda de 5 a 7 días en ser establecido, por lo tanto, se necesita de un puente inicial con heparina. Además, presentan múltiples interacciones con alimentos y medicamentos, y una estrecha necesidad de seguimiento. ${ }^{7,9,10}$

El procedimiento para determinar la intensidad del efecto anticoagulante de los AVK esta medido por el Índice Internacional Normalizado (INR), ${ }^{11}$ el INR en un paciente sano es de 0.9 a 1.6 , mientras que el rango útil ha sido reportado dependiendo de cada patología, así en pacientes con prótesis valvulares mecánicas y en tromboembolismo pulmonar con síndrome antifosfolipídico el INR es de 2.5 a 3.5; En tanto, la mayoría de pacientes con fibrilación auricular, accidente cerebrovascular isquémico, infarto agudo de miocardio y valvulopatías embolígenas el INR es de 2 a. ${ }^{12,31}$

Los ACDO simplifican y mejoran el manejo de pacientes en terapia anticoagulante, ya que actúan de manera específica sobre la cascada de coagulación, con una acción inmediata, una dosis fija y predecible, y escasa interacción con alimentos y otros fármacos. Los inhibidores directos de la trombina como el Dabigatrán y los inhibidores directos del factor Xa son el Rivaroxaban, Apixabán, Edoxabán y el Betrixabán. ${ }^{4,8,13,14}$

En los últimos tiempos, los pacientes que reciben terapia anticoagulante han aumentado, por lo tanto, en odontología es permitido el manejo bajo ciertas condiciones en las diferentes áreas, así tenemos que en cirugía oral es posible realizar una extracción dental de manera segura, $3,15,16$ teniendo en cuenta los efectos secundarios de los fármacos como una posible hemorragia post-extracción o en el caso de suspender el fármaco, la formación de un tromboembolismo. ${ }^{15,17}$

El presente estudio tiene como objetivo analizar mediante una revisión de la literatura el tipo de manejo que necesita un paciente anticoagulado, además de las limitaciones, indicaciones y medidas hemostáticas que debemos tomar en cuenta al momento de realizar una extracción dental en estos pacientes.

\section{Materiales y métodos}

En esta revisión bibliográfica se realizó una búsqueda en las bases digitales de Pubmed, Science Direct, Cochrane y SciELO considerando los artículos publicados en los últimos 5 años. En la estrategia de investigación se emplearon como árbol de búsqueda: (Warfarin) AND (Dental extraction); (Anticoagulant) AND (Dental extraction); (Direct oral anticoagulant) AND (Dental extraction); (Warfarin) AND (Dental surgery); (Anticoagulant) AND (Dental surgery); (Direct oral anticoagulant) AND (Dental surgery); (Warfarin) AND (Tooth extraction); (Anticoagulant) AND (Tooth extraction); y (Direct oral anticoagulant) AND (Tooth extraction)

Los criterios de inclusión para la selección de artículos fueron en el idioma inglés y español, accesibles en PDF, publicados desde el 2016 al 2020, sin tener en cuenta la edad y el sexo.

Luego de realizar la búsqueda en las bases de datos, se realizó un cribado aplicando los criterios a los 2176 artículos preseleccionados, donde solo 59 cumplieron con los criterios de inclusión y, por tanto, fueron analizados y discutidos.

Los artículos revisados fueron recolectados y almacenados usando Mendeley Desktop, que permite eliminar referencias bibliográficas duplicadas y como herramienta para la organización de los datos se empleó Microsoft Excel 2016. 


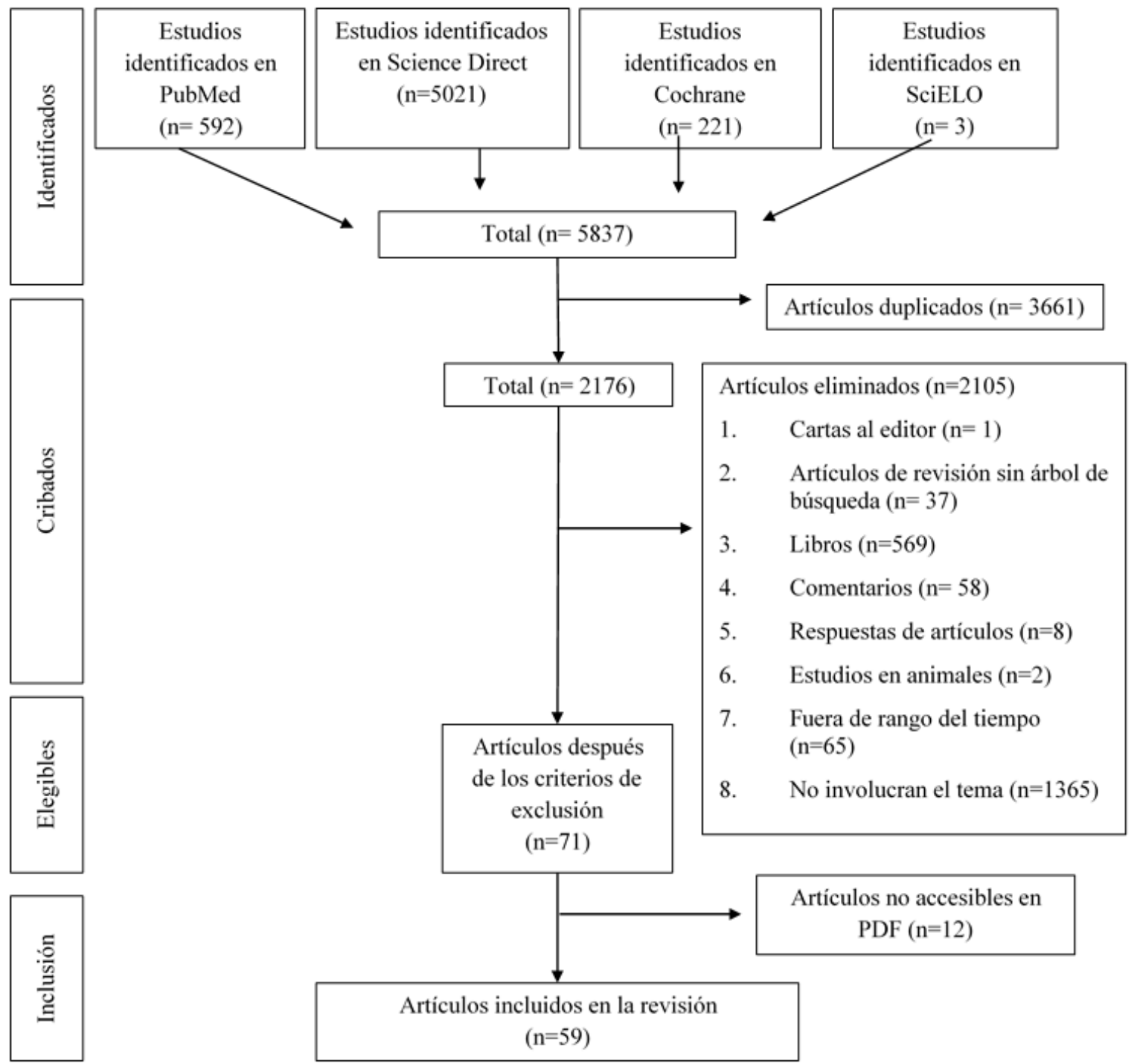

Fig. 1. Diagrama de flujo de selección de artículos de la revisión de literatura

\section{Resultados}

A lo largo de los años se han generado diferentes protocolos de cirugía dental para los pacientes anticoagulados, estos se modifican en relación con la terapia anticoagulante que reciben. ${ }^{18}$ Existen dos grupos de fármacos utilizados, así tenemos: los antagonistas de la vitamina $\mathrm{K}$ y los anticoagulantes orales directos. ${ }^{19}$

Al considerar el manejo de los pacientes bajo terapia anticoagulante, debemos tener en cuenta los riesgos que presentan a la hora de la extracción dental, que son:

1) Suspensión o reducción de la Terapia anticoagulante con riesgos de complicaciones embolicas.

2) Mantener la Terapia Anticoagulante con un mayor riesgo de hemorragia. ${ }^{20}$

En la actualidad se ha demostrado que existe un mayor riesgo para el paciente cuando se interrumpe la terapia anticoagulante porque puede producir tromboembolismo e "hipercoagulación de rebote", comparado con la hemorragia post-extracción al continuar con los anticoagulantes. ${ }^{2,20}$

La finalidad de los anticoagulantes es mantener todos los beneficios de la disminución del riesgo de tromboembolismo con un mínimo de efectos secundarios como hemorragias, 
hematomas, etc. ${ }^{1}$

Yagyuu et al. (2017) determinaron que no existe un consenso que nos asegure la atención de pacientes en donde se suspenda la toma de un medicamento ACDO y AVK al realizar los procedimientos de extracción dental. ${ }^{21}$

\subsection{Antagonistas de la vitamina $K(A V K)$}

A pesar de que los AVK presentan muchas desventajas como su ajuste de dosis, restricciones dietéticas y la necesidad de análisis de coagulación en sangre, uno de sus derivados, la Warfarina, sigue siendo la primera opción por su amplia disponibilidad, conveniencia de administración y rentabilidad. ${ }^{22,23}$

Los protocolos en pacientes sometidos a terapia con antagonistas de la vitamina K (AVK) durante las exodoncias son:

1) Suspender los AVK durante varios días

2) Suspender los AVK y administrar heparina antes del tratamiento dental

3) Reducir ( sin suspender) la terapia con AVK

4) Mantener la terapia AVK sin cambios y aplicar medidas hemostáticas locales ${ }^{2}$

\subsubsection{Suspender los antagonistas de la vitamina $K$ durante varios días}

Algunos autores propusieron suspender el tratamiento con anticoagulantes de 2 a 6 días antes de las extracciones, ya que se habían informado episodios hemorrágicos graves en varios casos. ${ }^{2}$

Además, la suspensión del AVK permite la normalización del INR y restablecimiento de la actividad de los factores de coagulación. ${ }^{24}$ Ahmed et al determinaron que después de 5 días de suspender el medicamento el INR bajo a 1.5 , lo cual nos lleva a un alto riesgo de tromboembolismo. ${ }^{2}$

\subsubsection{Suspender los antagonistas de la vitamina $K$ y admi- nistrar un puente de heparina antes del tratamiento dental}

La anticoagulación puente se refiere al uso de la heparina de bajo peso molecular (HBPM), que se administra por vía subcutánea en el momento de la interrupción de la warfarina. La reanudación de la warfarina se ajusta con la dosis preoperatoria regular hasta que el INR volviera al nivel terapéutico óptimo..$^{25,26}$

Algunos autores describen que se puede sustituir la warfarina por heparina antes de las extracciones dentales y luego continuarla después del tratamiento. ${ }^{2}$ Sin embargo, este procedimiento tiene un costo más alto, ya que, el paciente requiere un control cuidadoso del medicamento con atención especializada para administrarlo. ${ }^{2,19}$ Estos pacientes también tienen riesgo de tromboembolismo postoperatorio, puesto que la warfarina puede tardar varios días en alcanzar niveles terapéuticos. ${ }^{2,26}$

Cabbar et al. (2019) investigaron la necesidad del puente de heparina y sugirieron que causaba más hemorragia pero evitaba el riesgo de tromboembolismo. ${ }^{27}$ Mientras que, otros estudios en donde se compararon los diferentes protocolos, dio como resultado que no hay diferencias significativas en el sangrado posoperatorio. ${ }^{28}$

\subsubsection{Reducir (sin suspender) la terapia con antagonistas de la vitamina $K$}

Ahmed et al. (2019) comentaron que el protocolo de reducir la dosis antes de las extracciones dentales vuelve a producir que el paciente sea susceptible al riesgo de tromboembolismo, por lo que realizarlo tendrá un compromiso más alto. ${ }^{2}$ Sin embargo, no existe evidencia actual de este protocolo.

\subsubsection{Mantener la terapia antagonista de la vitamina $K$ sin cambios y aplicar medidas hemostáticas locales}

Los pacientes bajo terapia anticoagulante que desean realizarse cirugías menores, pueden mantener los medicamentos AVK si presentan las condiciones adecuados como son: INR seguro (3), planificación quirúrgica, cobertura antibiótica, técnicas atraumáticas, uso de agentes hemostáticos y cierre primario de la herida. ${ }^{29}$

Ciria et al. (2018) encontraron que el nivel de sangrado depende de los valores del INR, a medida que este valor aumenta, se amplía los niveles de sangrado1. Dando como resultado un INR de 1.0 a 1.99 un sangrado leve, INR 2.0 a 2.99 un sangrado leve en un $50 \%$ y en INR de 3.0 a 3.5 se presentó un sangrado moderado. ${ }^{1}$

Los AVK se pueden continuar con seguridad en procedimientos dentales de bajo riesgo siempre que el INR $\leq 3.5 .{ }^{30}$ La valoración del INR se debe realizar el mismo día de la cirugía oral y cuanto más elevado es el valor de INR mayor es el peligro de presentar una hemorragia 11,31. Los valores no solo nos ayudan a prevenir las complicaciones hemorrágicas, sino que también puede identificar desajustes 
de la dosis de AVK dando una percepción del estado actual del paciente. ${ }^{11}$

Rocha et al. (2019) realizaron una comparación entre pacientes que consumían AVK y pacientes sanos, en el cual se determinó que en los pacientes que se encontraban bajo terapia AVK, necesitaron un mayor número de medidas hemostáticas. $^{22}$

La warfarina suele combinarse con agentes antiplaquetarios, estos pacientes presentan una tasa muy alta de eventos hemorrágicos intracraneales, lo que sugiere que existe un mayor riesgo de una hemorragia post extracción en pacientes con terapia combinada. ${ }^{32}$

\subsection{Anticoagulantes directos orales (ACDO)}

En la actualidad al existir una variedad de medicamentos anticoagulantes se puede tomar la decisión de administrar un fármaco que nos ofrezca mayor seguridad, como es el caso de los anticoagulantes directos orales.

Los protocolos en pacientes sometidos a terapia con anticoagulantes directos orales (ACDO) durante las exodoncias son:

1) Suspender los ACDO un día antes o un día después del tratamiento quirúrgico

2) Mantener la terapia ACDO sin cambios y aplicar medidas hemostáticas locales

\subsubsection{Suspender los anticoagulantes directos orales un día} antes o un día después del tratamiento quirúrgico

El riesgo de que se produzca un cuadro tromboembólico aumenta a partir de al menos 72 horas de omitir la toma de ACDO, por lo tanto, determinaron que al realizar la intervención en estos pacientes se puede prescindir de la dosis matutina. ${ }^{33}$

Se ha demostrado que con el retiró de una dosis del medicamento un día antes o un día después de la intervención, los pacientes tuvieron un sangrado leve y no hubo un cambio significativo ${ }^{34,35}$ aunque se presentaba un sangrado tardío por el reinicio de la terapia anticoagulante. ${ }^{36}$

\subsubsection{Mantener la terapia de anticoagulante directos orales sin cambios y aplicar medidas hemostáticas locales}

Los ACDO son medicamentos seguros en relación al nivel de sangrado en las extracciones dentales simples, siempre y cuando no se interrumpa y se utilice medidas hemostáticas locales para prevenir la hemorragia postoperatorio. ${ }^{37-39} \mathrm{La}$ vida media plasmática de los ACDO varía de 7 a 17 horas, no se recomienda la suspensión de los ACDO en cirugías menores, en el caso de que la cirugía sea de alto riesgo se requiere interconsulta con el médico tratante para suspender la dosis 2 días antes de la cirugía. ${ }^{38}$

El manejo de ACDO para determinar el sangrado, no se da por los niveles del fármaco, sino por las propiedades farmacocinéticas del medicamento y el tipo de procedimiento quirúrgico. ${ }^{40}$ Se ha demostrado que no existe una diferencia significativa en la tasa de sangrado en los grupos ACDO,${ }^{41}$ pero Cocero et al. (2019) llegaron a la conclusión de que el sangrado excesivo en pacientes que toman ACDO se da por no respetar un intervalo entre la última ingesta y el momento de la cirugía, de al menos 4 a 6 horas y debe evitarse la extracción de dos o tres piezas contiguas en la misma cita (premolares y molares). ${ }^{42,43}$

El sangrado posoperatorio está relacionado con la extracción de múltiples piezas dentales en una sola cita, ${ }^{44}$ por lo tanto, si se desea extraer más dientes, se debe realizar interconsulta con el médico especialista para determinar el riesgo y beneficio de la intervención. ${ }^{45}$ Andrade et al (2018) encontraron que en los pacientes que consumen Dabigatrán presentan mayor frecuencia de sangrado 24 horas después de la extracción dental. ${ }^{46}$

\subsection{Protocolo para extracción en pacientes anticoagulados}

En los pacientes anticoagulados se necesita efectuar una exhaustiva investigación con el objetivo de saber el riesgo que corren al realizar una extracción dental, se debe tomar en cuenta la realización de una prueba de coagulación para conocer la probabilidad de sangrado luego de la exodoncia y además las medidas hemostáticas que se requiere para evitar una hemorragia. ${ }^{47,48}$

Hoy en día contamos con guías que nos permiten realizar de manera segura una cirugía en los pacientes bajo terapia anticoagulante. ${ }^{48}$ Sin embargo, se debe tomar en cuenta el tipo de medicamento que consume, se ha observado que el riesgo tromboembólico asociado a la suspensión del fármaco es menor en los $\mathrm{ACDO}$ en relación con los AVK. ${ }^{27}$ En esta revisión hemos recopilado los protocolos más utilizados para el manejo quirúrgico de pacientes anticoagulados.

\subsubsection{Antagonistas de la vitamina $K(A V K)$}

Debemos tener en cuenta que el paciente debe cumplir ciertos requisitos como son: ninguna patología asociada, INR $\leq 3.5$ tomado el mismo día de la cirugía y realizar 


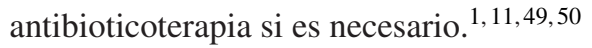

Si se cumple con las condiciones, se debe considerar que en la extracción de hasta 3 piezas dentales, no se recomienda el retiro del medicamento anticoagulante porque se puede controlar el sangrado realizando medidas hemostáticas. Si las extracciones son de 4 o más dientes, debemos contactar con el médico del paciente para determinar riesgos. ${ }^{11,39}$

\subsubsection{Anticoagulantes directos orales (ACDO)}

En pacientes que toman anticoagulantes orales directos se debe considerar el estado general del paciente mediante la observación de signos vitales y exámenes de laboratorio cómo un hemograma completo y revisar tiempo de coagulación como el tiempo de protrombina (TP) y tiempo de tromboplastina parcial TTP. ${ }^{37,51}$ Es necesario que exista un intervalo de 4 a 6 horas entre la última toma del medicamento y la cirugía. ${ }^{42}$

También se debe tomar en cuenta el número de piezas a extraer, si es menor a 3 la cirugía se puede ejecutar utilizando las medidas hemostáticas necesarias. Si debemos realizar exodoncias de 4 o más piezas dentarias, se recomienda contactar con el médico del paciente para determinar riesgos y beneficio que pueda conllevar la realización de la cirugía. $^{11,41}$

Tanto para los pacientes que se encuentran bajo terapia ACDO y AVK se debe seguir la evolución durante al menos 7 días y mantener una línea de emergencia las 24 horas con el objetivo de evitar complicaciones de hemorragia postoperatoria. ${ }^{13}$ Los odontólogos debemos ser cauteloso con las citas de seguimiento y comprometernos al bienestar del paciente. ${ }^{36}$

\subsubsection{Medidas hemostáticas para pacientes con terapia $A V K$ y $A C D O$}

El control de la hemorragia posquirúrgica es una preocupación común incluso en pacientes que no toman anticoagulantes y se someten a intervenciones menores. ${ }^{52}$

La hemorragia es una complicación postoperatoria que se puede predecir y minimizar tomando en cuenta la historia previa de pérdida de sangre y el patrón de sangrado perioperatoria. $^{22}$ Además, podemos aplicar medidas adicionales para conseguir una adecuada hemostasia, que son eficaces y nos ayudan en casos de sangrados persistentes. ${ }^{22,53,54} \mathrm{El}$ sangrado se clasifica según el nivel, así tenemos:
- Sangrado leve. - Es la pérdida de sangre manejable por el paciente.

- Sangrado moderado. - Es la pérdida prolongada de sangre que no puede ser manejada por el paciente.

- $\quad$ Sangrado severo.- Es la pérdida de sangre no manejable usando medidas hemostáticas, por lo que requieren terapia sistémica y/o hospitalización específica. ${ }^{42}$

Después de la extracción dental, el odontólogo debe aplicar medidas hemostáticas que van de acuerdo con la cantidad de sangrado de cada paciente y pueden ser combinados hasta que se detenga la hemorragia, ${ }^{35}$ así tenemos diferentes tipos de hemostasia que se logran aplicar:

1) Compresión local de la herida mordiendo una gasa durante $30 \mathrm{~min}$

2) Compresión local de la herida mordiendo una gasa empapado en un agente fibrinolítico durante 30 minutos

3) Insertar un relleno hemostático en el orificio de extracción como Oxicelulosa, Colágeno, Gelatina porcina

4) Electrocoagulación bipolar

5) Suturar con un material reabsorbible 4-0 y 5-02

Existen otros métodos capaces de generar hemostasia, los cuales no son muy utilizados, así tenemos:

- La compresión de las tablas óseas que rodean al diente y termoterapia fría, esta garantiza la formación del coágulo sanguíneo y vasoconstricción de la zona, previniendo la hemorragia. ${ }^{1}$

- Enjuague bucal con ácido tranexámico puede controlar el sangrado postoperatorio. ${ }^{55,56}$ Además, debe administrarse por 24 horas después de la extracción $\mathrm{y}$ en pacientes sometidos a terapia anticoagulante. ${ }^{57,58}$

- Coágulos de fibrina rica en plaquetas para minimizar la hemorragia post extracción dando resultados favorables a las 24 y 48 horas. $^{59}$

- Hemcon Dental Dressing (HDD) es un dispositivo medico hemostático que logra una hemostasia más corta (4 min.) y una buena cicatrización en cirugías orales menores. ${ }^{52}$

$\mathrm{AVK}=$ antagonistas de la vitamina $\mathrm{k} ; \mathrm{ACDO}=$ anticoa - 


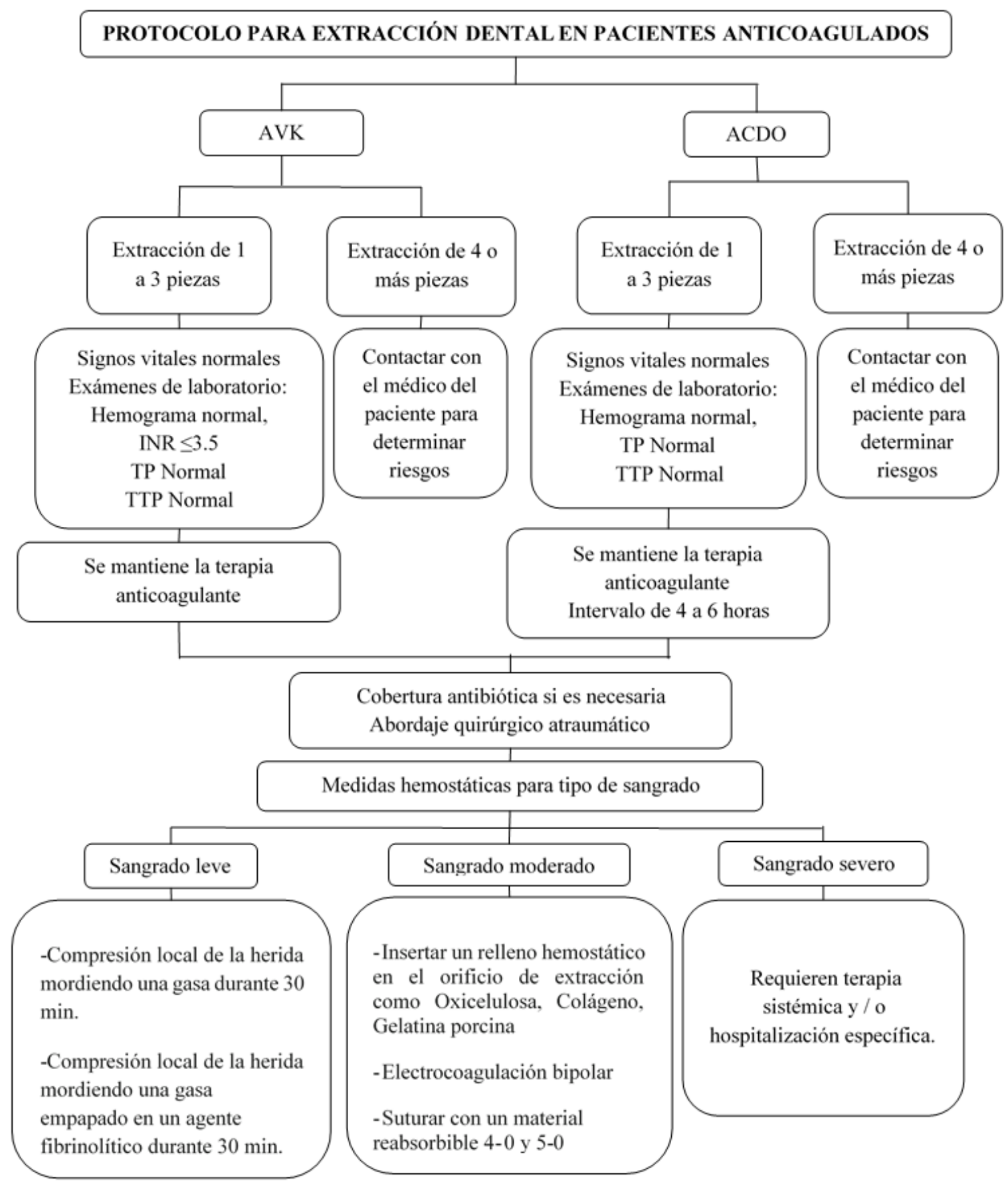

Fig. 2. Protocolo para extracción dental en pacientes anticoagulados

gulantes directos orales; INR= índice internacional normalizado; $\mathrm{TP}=$ tiempo de protrombina; $\mathrm{TTP}=$ tiempo de tromboplastina parcial

\section{Conclusiones}

Dada la amplia heterogeneidad metodológica de los estudios procesados en esta revisión hemos encontrado que se necesitan más ensayos clínicos que empleen una metodología similar y con mayor tamaño muestral. Sin embargo, en función de nuestro análisis podemos informar 
que en los pacientes sometidos a terapia anticoagulante con Antagonistas de la vitamina $\mathrm{K}$ no se debe retirar o modificación la toma del medicamento, el INR tiene que ser $\leq 3.5$, la extracción de 1 a 3 piezas dentarias es segura y debemos emplear una buena planificación quirúrgica con técnicas atraumáticas y el uso de medidas hemostáticas adecuadas al tipo de sangrado.

En cambio, los pacientes bajo la terapia con Anticoagulantes directos orales nos brindan una mayor seguridad, al respetar un intervalo de 4 a 6 horas entre la última toma del medicamento y la cirugía dental, sin necesidad de retirar ni modificar las tomas del fármaco y con la exodoncia atraumática de máximo 3 piezas dentarias, haciendo fácil conllevar un sangrado capaz de controlarse mediante medidas hemostáticas.

Es fundamental para el odontólogo tener el conocimiento actualizado del trato a pacientes anticoagulados y sus complicaciones hemorrágicas, además, 10 de conocer los costos que tienen las medidas hemostáticas y su adecuado uso.

Conflicto de intereses: Los autores del presente estudio manifiestan que no existe ningún conflicto de intereses en relación al tema de estudio.

\section{Referencias}

1 González CBC, Mera AMP, Roch ETC, González LE, Millián MB. Extracciones dentarias sin modificar la terapia anticoagulante en pacientes con enfermedad cardiovascular. Revista Cubana de Cardiología y Cirugía Cardiovascular. 2018;24(3).

2 Ahmed I, Younis M, Shah AA. Extraction in Patients on Oral Anticoagulant Therapy With and without Stopping the Drug: A Comparative Study. Journal of Maxillofacial and Oral Surgery. 2019;18(4):555-558.

3 Motta RHL, Bergamaschi CDC, De Andrade NK, Guimaraes CC, Ramacciato JC, Araújo JDO, et al. Bleeding risk in patients using oral anticoagulants submitted to surgical procedures in dentistry: A systematic review protocol. BMJ Open [Internet]. diciembre de 2017;7(12). Disponible en: https://pubmed.ncbi.nlm.nih.gov/29259064/

4 Berton F, Costantinides F, Rizzo R, Franco A, Contarin J, Stacchi C, et al. Should we fear direct oral anticoagulants more than vitamin $\mathrm{K}$ antagonists in simple single tooth extraction? A prospective comparative study. Clinical Oral Investigations. agosto de 2019;23(8):3183-3192.

5 Rubino RT, Dawson DR, Kryscio RJ, Al-Sabbagh M, Miller CS. Postoperative bleeding associated with antiplatelet and anticoagulant drugs: A retrospective study. Oral Surgery, Oral Medicine, Oral Pathology and Oral Radiology. septiembre de 2019;128(3):243-9.
6 Shi Q, Xu J, Zhang T, Zhang B, Liu H. Postoperative Bleeding Risk in Dental Surgery for Patients on Oral Anticoagulant Therapy: A Meta-analysis of Observational Studies. Frontiers in Pharmacology. febrero de 2017;08(FEB):58.

7 Bensi C, Belli S, Paradiso D, Lomurno G. Postoperative bleeding risk of direct oral anticoagulants after oral surgery procedures: a systematic review and meta-analysis. International Journal of Oral and Maxillofacial Surgery. 1 de julio de 2018;47(7):923-32.

8 Manfredi M, Dave B, Percudani D, Christoforou J, Karasneh J, Diz Dios P, et al. World workshop on oral medicine VII: Direct anticoagulant agents management for invasive oral procedures: A systematic review and meta-analysis. Oral Diseases. junio de 2019;25(S1):157-173.

9 ichi Yamada S, Hasegawa T, Soutome S, Yoshimura H, Miyakoshi M, Ueda N, et al. Prevalence of and risk factors for postoperative hemorrhage after lower third molar extraction on warfarin therapy: a multicenter retrospective study in Japan. Odontology. 2020;108(3):462-469.

10 Molina Castaño D, Campos Quesada M, Núñez Guerrero A. Historia de los anticoagulantes y su uso clínico en el presente. Rev.méd.sinerg. 1 de febrero de 2020;5(2):e365.

11 Lombardi N, Varoni EM, Sorrentino D, Lodi G. International normalized ratio (INR) values in patients receiving oral vitamin $\mathrm{K}$ antagonists and undergoing oral surgery: A clinical audit. Spec Care Dentist. julio de 2020;40(4):37481.

12 Nieves Bel Peña. Guía para el seguimiento Farmacológico Individualizado en Personas con Tratamiento de Anticoagulación Oral (v.2).

13 Hanken H, Gröbe A, Heiland M, Smeets R, Kluwe L, Wikner J, et al. Postoperative bleeding risk for oral surgery under continued rivaroxaban anticoagulant therapy. Clin Oral Investig. julio de 2016;20(6):1279-82.

14 Yang S, Shi Q, Liu J, Li J, Xu J. Should oral anticoagulant therapy be continued during dental extraction? A metaanalysis. BMC Oral Health. 2016;16(1):1-9.

15 Ruiz Gutiérrez MF, Oliva Mella P, Universidad del Desarrollo, Universidad del Desarrollo. Manejo de pacientes con terapia anticoagulante sometidos a extracciones dentales: una revisión sistemática. Rev Fac Odontol Univ Antioq [Internet]. febrero de 2016 [citado 18 de septiembre de 2020];27(2). Disponible en: https://revistas.udea.edu.co/index.php/odont/article/view/16368

16 Inokoshi M, Kubota K, Yamaga E, Ueda K, Minakuchi S. Postoperative bleeding after dental extraction among elderly patients under anticoagulant therapy. Clin Oral Invest [Internet]. 1 de septiembre de 2020 [citado 19 de septiembre de 2020]; Disponible en: http://link.springer.com/10.1007/s00784-020-03559-z

17 Morimoto Y, Yokoe C, Imai Y, Sugihara M, Futatsuki $\mathrm{T}$. Tooth extraction in patients taking nonvitamin $\mathrm{K}$ antagonist oral anticoagulants. Journal of Dental Sciences. 2016;11(1):59-64. 
18 Rai S, Rattan V. Efficacy of Feracrylum as Topical Hemos- 30 Biedermann JS, Rademacher WMH, Hazendonk HCAM, tatic Agent in Therapeutically Anticoagulated Patients Undergoing Dental Extraction: A Comparative Study. Journal of Maxillofacial and Oral Surgery. 2019;18(4):579-583.

19 Lukacs D, Staczer N, Vajta L, Olasz L, Joob-Fancsaly A, Szalma J. Dental and Oral Surgical Treatment of 31 Medication-Induced Bleeding Patients: Audit of the National Guideline in Hungary. Orvosi Hetilap. octubre de 2016;157(43):1722-1728. van Diermen DE, Leebeek FWG, Rozema FR, et al. Predictors of oral cavity bleeding and clinical outcome after dental procedures in patients on vitamin $\mathrm{K}$ antagonists. A cohort study. Thromb Haemost. 27 de 2017;117(7):1432-9.

1 Febbo A, Cheng A, Stein B, Goss A, Sambrook P. Postoperative Bleeding Following Dental Extractions in Patients Anticoagulated With Warfarin. Journal of Oral and Maxillofacial Surgery. agosto de 2016;74(8):1518-1523.

20 Schmitt C, Rusche B, Clemm R, Neukam F, Buchbender 32 Kataoka T, Hoshi K, Ando T. Is the HAS-BLED score M. Management of anticoagulated patients in dentoalveolar surgery: a clinical comparative study. Clinical Oral Investigations. noviembre de 2019;1-10.

21 Yagyuu T, Kawakami M, Ueyama Y, Imada M, Kurihara 33 M, Matsusue Y, et al. Risks of postextraction bleeding after receiving direct oral anticoagulants or warfarin: A retrospective cohort study. BMJ Open. 2017;7(8):1-8.

22 Rocha AL, Oliveira SR, Souza AF, Travassos DV, Abreu LG, Ribeiro DD, et al. Bleeding assessment in oral surgery: 34 A cohort study comparing individuals on anticoagulant therapy and a non-anticoagulated group. Journal of CranioMaxillofacial Surgery. 1 de mayo de 2019;47(5):798-804. useful in predicting post-extraction bleeding in patients taking warfarin? A retrospective cohort study. BMJ Open. 2016;6(3).

33 Miclotte I, Vanhaverbeke M, Agbaje JO, Legrand P, Vanassche T, Verhamme $\mathrm{P}$, et al. Pragmatic approach to manage new oral anticoagulants in patients undergoing dental extractions: a prospective case-control study. Clin Oral Investig. septiembre de 2017;21(7):2183-8.

34 Patel JP, Woolcombe SA, Patel RK, Obisesan O, Roberts $\mathrm{LN}$, Bryant $\mathrm{C}$, et al. Managing direct oral anticoagulants in patients undergoing dentoalveolar surgery. British Dental Journal. febrero de 2017;222(4):245-249.

23 Hakata K, Okubo M, Hasegawa T, Izumi S, Komiyama 35 Lanau N, Mareque J, Giner L, Zabalza M. Direct oral $\mathrm{Y}$, Uchida D, et al. Alterations of serum vitamin $\mathrm{K}$ levels and prothrombin time-international normalized ratio during tooth extractions among patients receiving warfarin. Journal 36 of Oral and Maxillofacial Surgery, Medicine, and Pathology. noviembre de 2018;30(6):550-553.

24 Clark NP, Douketis JD, Hasselblad V, Schulman S, Kindzelski AL, Ortel TL. Predictors of perioperative major bleeding in patients who interrupt warfarin for an elective surgery or procedure: Analysis of the BRIDGE trial. American Heart Journal. enero de 2018;195:108-14.

anticoagulants and its implications in dentistry. A review of literature. J Clin Exp Dent. 2017;0-0.

Müller M, Schlittler F, Schaller B, Nagler M, Exadaktylos AK, Sauter TC. Characteristics, treatment and outcome of bleeding after tooth extraction in patients on DOAC and phenprocoumon compared to non-anticoagulated patients-a retrospective study of emergency department consultations. Clinical Oral Investigations. mayo de 2019;23(5):2273-2278.

25 Erden İ, Erden EÇ, Aksu T, Gölcük ŞE, Turan B, Erkol A, et al. Comparison of uninterrupted warfarin and bridging therapy using low-molecular weight heparin with respect to the severity of bleeding after dental extractions in patients with prosthetic valves. Anatolian Journal of Cardiology. 38 2016;16(7):467-473.

26 Lu SY, Lin LH, Hsue SS. Management of dental extractions in patients on warfarin and antiplatelet therapy. Journal of the Formosan Medical Association. 2018;117(11):979-986.

27 Cabbar F, Cabbar AT, Coşansu K, Çekirdekçi Eİ. Ef- 3 fects of Direct Oral Anticoagulants on Quality of Life During Periprocedural Management for Dental Extractions. Journal of Oral and Maxillofacial Surgery. mayo de 2019;77(5):904-911.

28 Lee J-S, Kim M-K, Kang S-H. Effect of warfarin discontinuation on the incidence of postoperative bleeding in tooth extraction. JKAOMS. 31 de agosto de 2020;46(4):228-34.

37 Caliskan M, Tükel HC, Benlidayi ME, Deniz A. Is it necessary to alter anticoagulation therapy for tooth extraction in patients taking direct oral anticoagulants? Medicina Oral, Patologia Oral y Cirugia Bucal. noviembre de 2017;22(6):e767-e773.

38 Mauprivez C, Khonsari RH, Razouk O, Goudot P, Lesclous $\mathrm{P}$, Descroix V. Management of dental extraction in patients undergoing anticoagulant oral direct treatment: a pilot study. Oral Surgery, Oral Medicine, Oral Pathology and Oral Radiology. noviembre de 2016;122(5):e146-e155.

39 Kwak EJ, Nam S, mee Park K, yul Kim S, Huh J, Park W. Bleeding related to dental treatment in patients taking novel oral anticoagulants (NOACs): a retrospective study. Clinical Oral Investigations. enero de 2019;23(1):477-484.

40 Brennan Y, Gu Y, Schifter M, Crowther H, Favaloro EJ, Curnow J. Dental extractions on direct oral anticoagulants vs. warfarin: The DENTST study. Research and Practice in Thrombosis and Haemostasis. febrero de 2020;4(2):278-284.

29 Daroit NB, Festugatto FL, Beltrame LF, Maciel JCC, Rados 41 Lababidi E, Breik O, Savage J, Engelbrecht H, Kumar R, PV. Analysis of profiles of patients on oral anticoagulants undergoing dental extraction - A retrospective study. JORDI - Journal of Oral Diagnosis. 2017;2(1):1-5. Crossley CW. Assessing an oral surgery specific protocol for patients on direct oral anticoagulants: a retrospective controlled cohort study. Int J Oral Maxillofac Surg. julio de 
2018;47(7):940-6.

42 Cocero N, Basso M, Grosso S, Carossa S. Direct Oral Anticoagulants and Medical Comorbidities in Patients Needing Dental Extractions: Management of the Risk 53 of Bleeding. Journal of Oral and Maxillofacial Surgery. 2019;77(3):463-470.

43 Yoshikawa H, Yoshida M, Yasaka M, Yoshida H, Murasato Y, Fukunaga D, et al. Safety of tooth extraction in patients receiving direct oral anticoagulant treatment versus warfa- 54 rin: a prospective observation study. Int J Oral Maxillofac Surg. agosto de 2019;48(8):1102-8.

44 Hasegawa T, Yanamoto S, Tachibana A, Kojima Y, Koyama Y, Maeda M, et al. The risk factors associated 55 with postoperative hemorrhage after tooth extraction: a multi-center retrospective study of patients receiving oral antithrombotic therapy. Oral and Maxillofacial Surgery. 2017;21(4):397-404.

45 Ehrhard S, Burkhard JP, Exadaktylos AK, Sauter TC. Severe Enoral Bleeding with a Direct Oral Anticoagulant after 56 Tooth Extraction and Heparin Bridging Treatment. Case Reports in Emergency Medicine. 2019;2019:1-5.

46 Andrade MVS, Andrade LAP, Bispo AF, Freitas L de A, Andrade MQS, Feitosa GS, et al. Evaluation of the bleeding intensity of patients anticoagulated with warfarin or dabiga- 57 tran undergoing dental procedures. Arquivos Brasileiros de Cardiologia. 2018;111(3):394-399.

47 de Vasconcellos SJ de A, de Santana Santos T, Reinheimer DM, Faria-e-Silva AL, de Melo M de FB, Martins-Filho PRS. Topical application of tranexamic acid in anticoa- 58 gulated patients undergoing minor oral surgery: A systematic review and meta-analysis of randomized clinical trials. Journal of Cranio-Maxillofacial Surgery. enero de 2017;45(1):20-6.

48 Suresh V, Bishawi M, Manning MW, Patel C, Rogers J, Mi- 59 lano C, et al. Management of Patients With Left Ventricular Assist Devices Requiring Teeth Extraction: Is Halting Anticoagulation Appropriate? Journal of Oral and Maxillofacial Surgery. 2018;76(9):1859-1863.

49 Dudek D, Marchionni S, Gabriele M, Iurlaro A, Helewski $\mathrm{K}$, Toti $\mathrm{P}$, et al. Bleeding Rate After Tooth Extraction in Patients Under Oral Anticoagulant Therapy. Journal of Craniofacial Surgery. julio de 2016;27(5):1228-1233.

50 Cascalho Serra I da C, Ribeiro L da CAN, Gemito MLGP, Mendes FRP. Therapeutic management of users with oral anticoagulant therapy. eglobal. 9 de enero de 2016;15(1):10.

51 Muñoz-Corcuera M, Ramírez-Martínez-Acitores L, LópezPintor R-M, Casañas-Gil E, Hernández-Vallejo G. Dabigatran: A new oral anticoagulant. Guidelines to follow in oral surgery procedures. A systematic review of the literature. Medicina oral, patologia oral y cirugia bucal. noviembre de 2016;21(6):e679-e688.

52 Kumar KRA, Kumar J, Sarvagna J, Gadde P, Chikkaboriah S. Hemostasis and Post-operative Care of Oral Surgical Wounds by Hemcon Dental Dressing in Patients on Oral
Anticoagulant Therapy: A Split Mouth Randomized Controlled Clinical Trial. J Clin Diagn Res. septiembre de 2016;10(9):ZC37-40.

53 Zirk M, Fienitz T, Edel R, Kreppel M, Dreiseidler T, Rothamel D. Prevention of post-operative bleeding in hemostatic compromised patients using native porcine collagen fleeces-retrospective study of a consecutive case series. Oral and Maxillofacial Surgery. 2016;20(3):249-254.

54 Sohn JB, Lee H, Han YS, Jung DU, Sim HY, Kim HS, et al. When do we need more than local compression to control intraoral haemorrhage? Journal of the Korean Association of Oral and Maxillofacial Surgeons. 2019;45(6):343-350.

5 Ockerman A, Vanhaverbeke M, Miclotte I, Belmans A, Vanassche T, Politis $\mathrm{C}$, et al. Tranexamic acid to reduce bleeding after dental extraction in patients treated with nonvitamin $\mathrm{K}$ oral anticoagulants: design and rationale of the EXTRACT-NOAC trial. British Journal of Oral and Maxillofacial Surgery. 2019;57(10):1107-1112.

6 Engelen ET, Schutgens RE, Mauser-Bunschoten EP, van Es RJ, van Galen KP. Antifibrinolytic therapy for preventing oral bleeding in people on anticoagulants undergoing minor oral surgery or dental extractions. Cochrane Database Syst Rev. 02 de 2018;7:CD012293.

57 Queiroz SIML, Silvestre VD, Soares RM, Campos GBP, Germano AR, da Silva JSP. Tranexamic acid as a local hemostasis method after dental extraction in patients on warfarin: A randomized controlled clinical study. Clinical Oral Investigations. 2018;22(6):2281-2289.

58 Owattanapanich D, Ungprasert P, Owattanapanich W. Efficacy of local tranexamic acid treatment for prevention of bleeding after dental procedures: A systematic review and meta-analysis. Journal of Dental Sciences. marzo de 2019;14(1):21-6.

de Almeida Barros Mourão CF, Miron RJ, de Mello Machado RC, Ghanaati S, Alves GG, Calasans-Maia MD. Usefulness of platelet-rich fibrin as a hemostatic agent after dental extractions in patients receiving anticoagulant therapy with factor Xa inhibitors: a case series. Oral and Maxillofacial Surgery. septiembre de 2019;23(3):381-386.

Recibido: 01 de marzo de 2021

Aceptado: 12 de abril de 2021 